\title{
Feature-based processing of personally familiar faces in prosopagnosia: Evidence from eye-gaze contingency
}

\author{
Goedele Van Belle ${ }^{\mathrm{a}, *}$, Philippe Lefèvre ${ }^{\mathrm{a}}$, Renaud Laguesse ${ }^{\mathrm{a}}$, Thomas Busigny ${ }^{\mathrm{a}}$, Peter de Graef ${ }^{\mathrm{b}}$, \\ Karl Verfaillie ${ }^{\mathrm{b}}$ and Bruno Rossion ${ }^{\mathrm{a}}$ \\ ${ }^{\text {a } U n i v e r s i t e ́ ~ C a t h o l i q u e ~ d e ~ L o u v a i n, ~ L o u v a i n-l a-N e u v e, ~ B e l g i u m ~}$ \\ ${ }^{\mathrm{b}}$ Katholieke Universiteit Leuven, Leuven, Belgium
}

\section{Introduction}

How familiar and unfamiliar faces are perceived remains largely unknown. Two views have dominated this field of research. On the one hand, recordings of eye fixations on faces [10] and response classification experiments [2] suggest that a face is processed in terms of its individual components, or facial features (mouth, eyes, nose,...), a strategy called analytical processing. On the other hand, there is strong behavioral evidence for interdependence in the processing of different features of a face [6,7], rather supporting holistic processing of the face [7]. According to the latter holistic view, facial features are simultaneously perceived and integrated into a single representation, so that the perceptual field is that of the whole face.

To shed light on this issue, in two recent studies, we recorded eye movements in a neurological patient [5] suffering from a selective impairment in face recognition (acquired prosopagnosia). Previously, we showed that (1) PS fixates exactly on each of the main features of the face (mouth, left eye, right eye), contrary to normal observers who fixate mainly centrally on the top of the nose, around the geometric centre of the face [3]

*Corresponding author: Goedele van Belle, Université Catholique de Louvain (UCL), Faculté de Psychologie et des Sciences de l'Education (PSP), Unité de Cognition et Développement (CODE), Place du Cardinal Mercier, 10, B-1348 Louvain-la-Neuve, Belgium, Tel.: +32 104792 60; Fax: +32 104737 74; E-mail: goedele. vanbelle@uclouvain.be.
(Fig. 1a). Moreover (2), an original gaze-contingent stimulus presentation method [9] applied to an unfamiliar face discrimination task led us to demonstrate that, contrary to normal observers, PS' perceptual field appears to be limited to one central feature fixated at a time [8] (Fig. 1b). These observations indicate that prosopagnosia prevents processing the multiple elements of a whole face simultaneously, and thus that this ability is a key aspect in human face recognition expertise. Here, we extend these observations by testing the same patient with eye gaze contingency while she attempts to identify a large set of personally familiar individuals from their face.

\section{Methods}

PS (born 1950) had brain damage in 1992, with a main lesion in the right inferior occipital cortex. Difficulty at identifying faces is her only complaint, and object recognition is preserved. She was tested in numerous behavioural and neuroimaging studies over the past 10 years (see e.g. [1,5]). Despite her prosopagnosia, she still works as a kindergarten teacher, so that she is familiar with a large set of young children and uses many cues to recognize them in everyday life (3-4 years old; see [3] for details).

Here we took high quality photographs of 29 of these children's faces (2009). Extrafacial features were cropped. Stimuli were displayed using Presentation, on a 22 " Sony Trinitron monitor at a viewing distance 


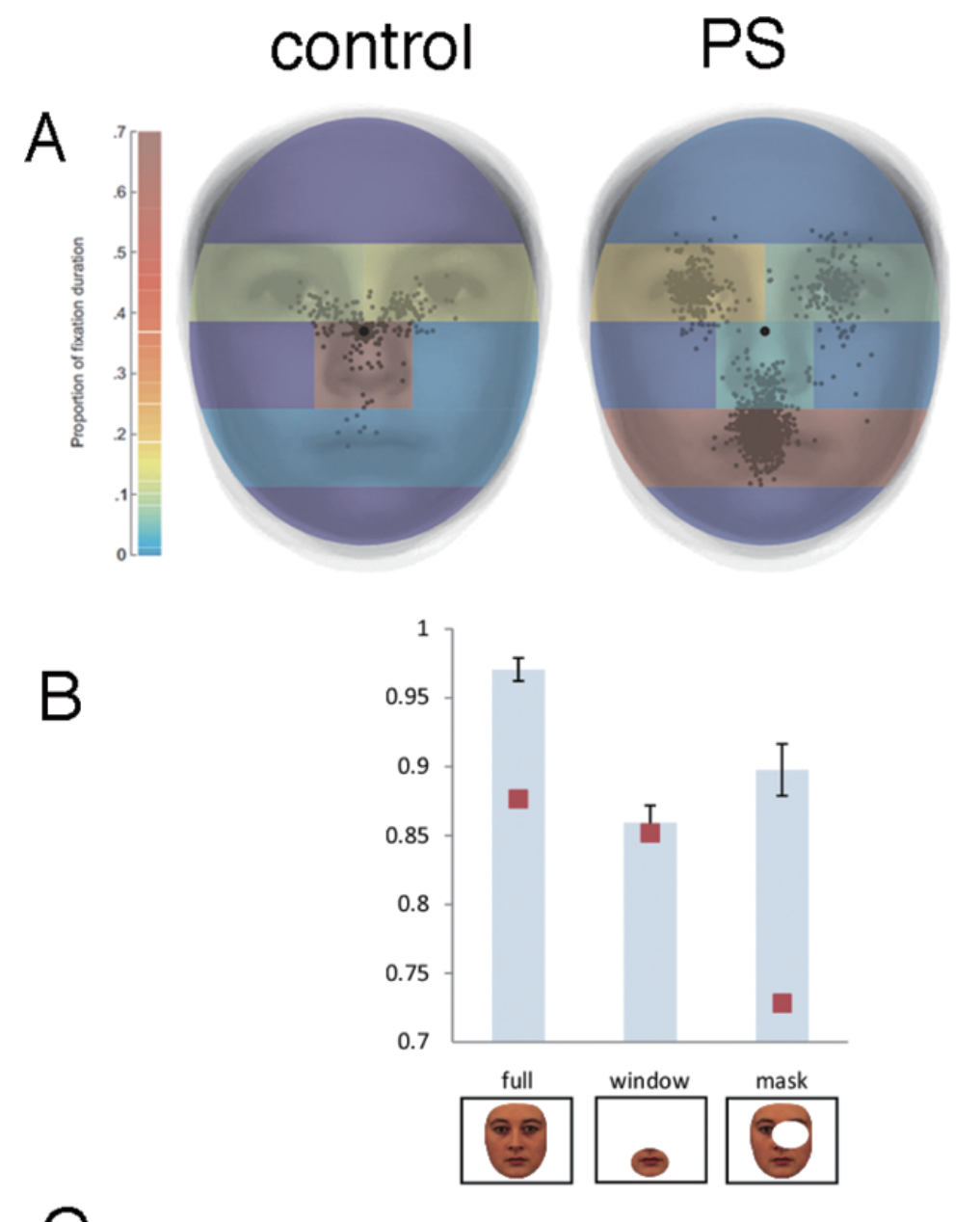

Q

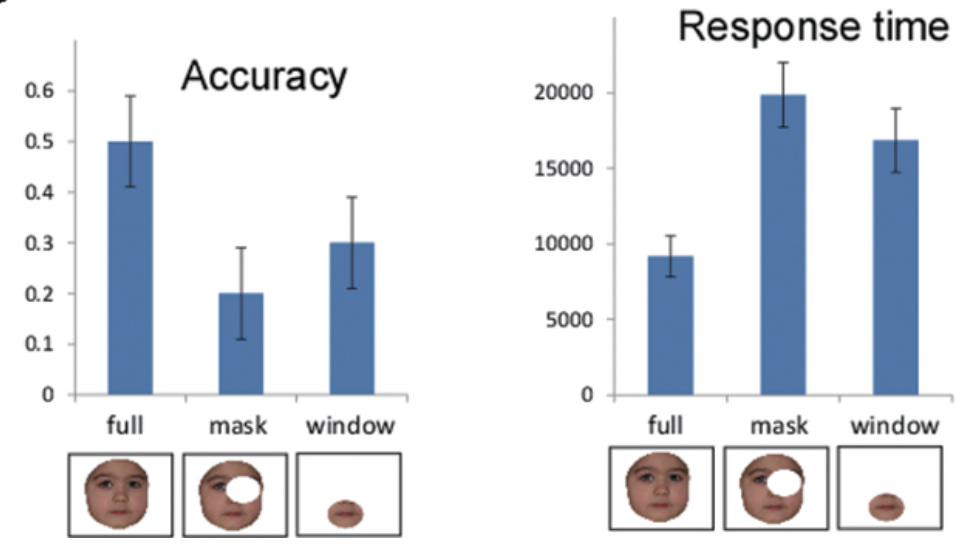

Fig. 1. A. Adapted from Orban de Xivry et al. [3]: fixation location of a normal observer and PS when identifying personally familiar faces. Note that PS fixates exactly on each diagnostic feature, with a very large proportion of fixations on the mouth (about $60 \%$ ). B. Accuracy results of the eye-gaze contingency study of Van Belle et al. [8], showing that the prosopagnosic patient PS' performance (the square dots) is much more affected in the mask than in the window condition, contrary to normal observers (the bars). C. In the present short study, gaze contingency was used with pictures of personally familiar children for the patient PS, who performed again lower with a window than a mask (increase of errors ad RTs; accuracy level is based on an identification task with a chance level of 1/29). 
of $58 \mathrm{~cm}$ with a spatial resolution of 1280 by 1024 pixels and a refresh rate of $80 \mathrm{~Hz}$. Height of the faces was 12 degrees of visual angle. In addition to the full view condition, faces could be presented with either a gaze-contingent window or a gaze contingent mask (Fig. 1B and 1C). A window revealed only the momentarily fixated feature of the stimulus, forcing PS to use an analytical processing strategy (one feature at a time). A gaze-contingent mask, on the contrary, constantly covered the centre of gaze, containing the most detailed, high-resolution information and therefore interfered with an analytical processing strategy, forcing PS to try to rely on holistic processing. The size of the mask and window was 8.5 by 6.5 degrees of vision.

Both stimulus display and response registration were handled by an Intel Centrino vPro. Eye movements were registered with an SR Research Eyelink 1000 remote eye tracker at a sampling rate of $250 \mathrm{~Hz}$ and with gaze position error smaller than $0.5^{\circ}$. Head movement was restricted by a chin and head rest.

A drift correction was followed by a fixation cross to the left of the face area. Upon fixation of the cross, the face of one of the children appeared, visible through one of the three viewing conditions. PS could freely explore the face and hit the space bar as soon as she thought she could identify the child. Then a list of all names appeared and PS responded by simultaneously fixating the recognized expression and hitting the space bar a second time. Accuracy rates, exploration duration (ms), number of saccades and fixations were measured.

PS completed 2 blocks of 45 trials each. Viewing conditions were randomized within the blocks.

\section{Results and discussion}

PS' level of performance with faces presented in full view was at $50 \%$, so she recognized half of the children from their (cropped) faces (chance level is 1/29). This performance dropped in the two gaze-contingent conditions, to $30 \%$ in the window condition (statistically lower than full view condition: $\mathrm{X}^{2}$ test of two proportions, $p=0.06$ ) and $20 \%$ in the mask condition ( $p=$ $0.009)$. The mask and window condition did not differ significantly from each other $(p=0.18)$. PS was slower, made more fixations and saccades in the two gazecontingent conditions than when the faces were presented at full view (Fig. 1C; full vs. window or full vs. mask, all ps $<0.001)$. However, in line with accuracy scores, she was also marginally slower in the mask than in the window condition $(p=0.15)$ and made more and longer fixations ( $p=0.07$ for both measures). We were not able to test normal controls in this task, given that PS was the only person who had been exposed to this large set of children faces and was available for the experiment. Nevertheless, these results are in line with observations made on the same patient when matching unfamiliar faces with gaze-contingency [8] (Fig. 1B). In that study, normal observers presented an opposite response profile than the patient, performing better in the mask than in the window condition. Moreover, a recent study with normal observers and familiar faces also show that identifying faces is generally faster in the mask than in the window condition (Ramon et al., submitted), unlike what is found for PS here.

Altogether, these observations support the view that the prosopagnosic patient PS has difficulties with processing the whole of an individual face, and that she rather relies on individual facial features in order to identify faces. Hence, being able to process an individual face holistically appears to be a key element in human face perception expertise.

\section{References}

[1] T. Busigny and B. Rossion, Acquired prosopagnosia abolishes the face inversion effect, Cortex 46 (2010), 965-981.

[2] N.D. Haig, How faces differ - a new comparative technique, Perception 14 (1985), 601-615.

[3] J.-J. Orban de Xivry, M. Ramon, P. Lefèvre and B. Rossion, Reduced fixation on the upper area of personally familiar faces following acquired prosopagnosia, Journal of Neuropsychology 2 (2008), 245-268.

[4] M. Ramon, G. Van Belle, P. Lefèvre and B. Rosion, (submitted). Holistic perception of fa, miliar and unfamiliar afces: an eye-gaze contingency study.

[5] B. Rossion, R. Caldara, M. Seghier, A.-M. Schuller F. Lazeyras and E. Mayer, A network of occipito-temporal facesensitive areas besides the right middle fusiform gyrus is necessary for normal face processing, Brain 126 (2003), 23812395.

[6] J. Sergent, An investigation into component and configural processes underlying facial perception, British Journal of Psychology 75 (1984), 221-242.

[7] J.W. Tanaka and M.J. Farah, Parts and wholes in face recognition. The Quarterly Journal of Experimental Psychology. A, Human Experimental Psychology 46 (1993), 225-245.

[8] G. Van Belle, P. de Graef, K. Verfaillie, T. Busigny and B. Rossion, Whole not hole: expert face recognition requires holistic perception, Neuropsychologia 48 (2010), 2609-2620.

[9] P.M.J. van Diepen, P. De Graef and J. Van Rensbergen, On-line control of moving masks and windows on a complex background using the ATVista videographics adapter, Behavior Research Methods, Instruments, and Computers 26 (1994), 454-460.

[10] A.L. Yarbus, Eye movements during perception of complex objects, in Eye Movements and Vision, L.A. Riggs, ed., Plenum Press, New York, 1967. 


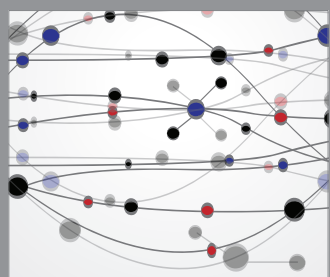

The Scientific World Journal
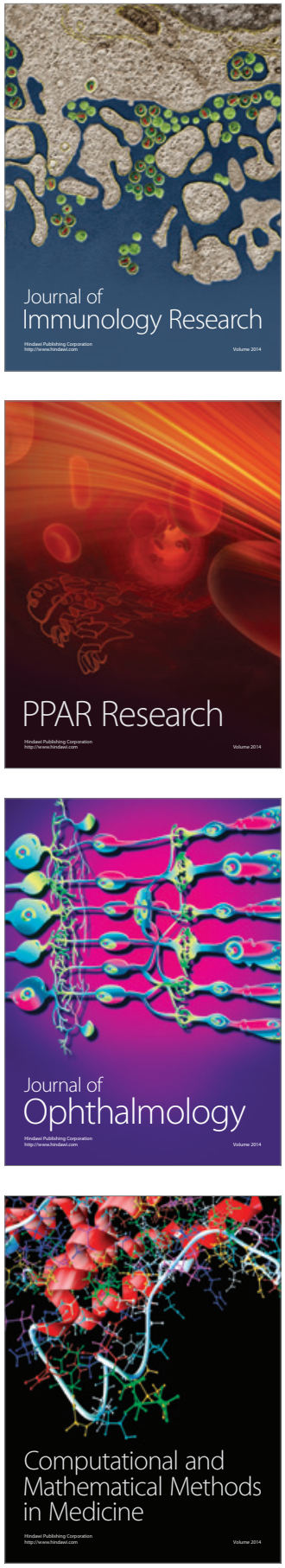

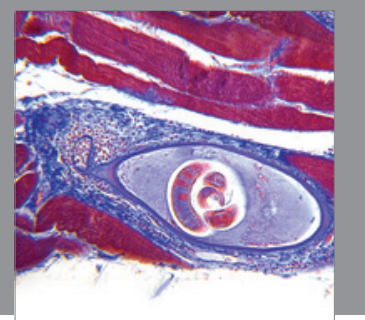

Gastroenterology

Research and Practice
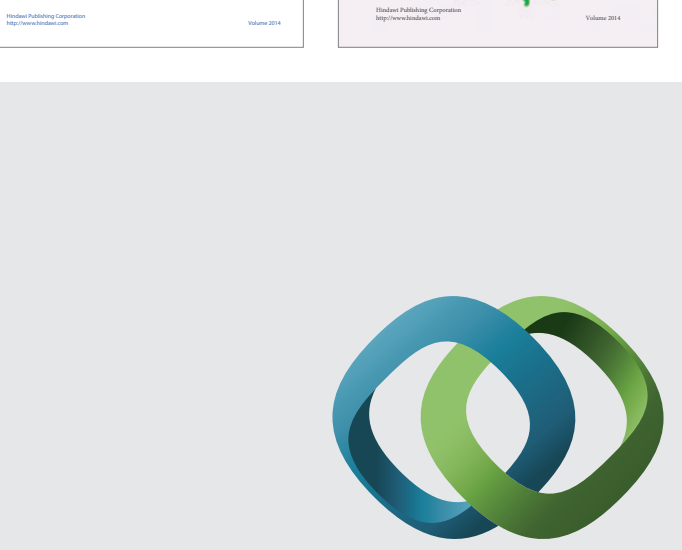

\section{Hindawi}

Submit your manuscripts at

http://www.hindawi.com
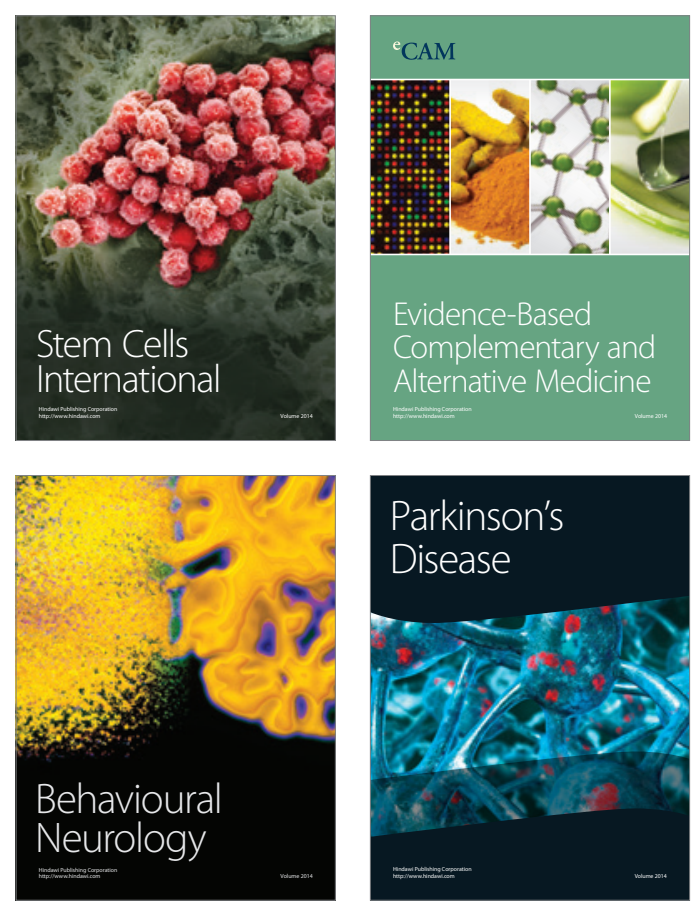

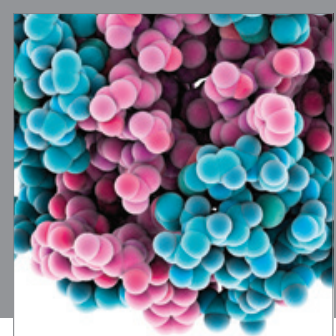

Journal of
Diabetes Research

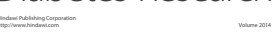

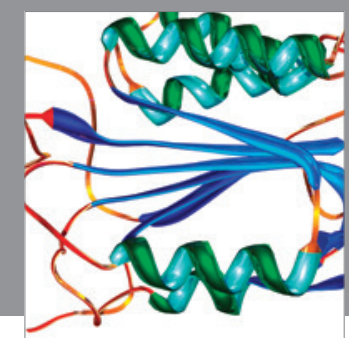

Disease Markers
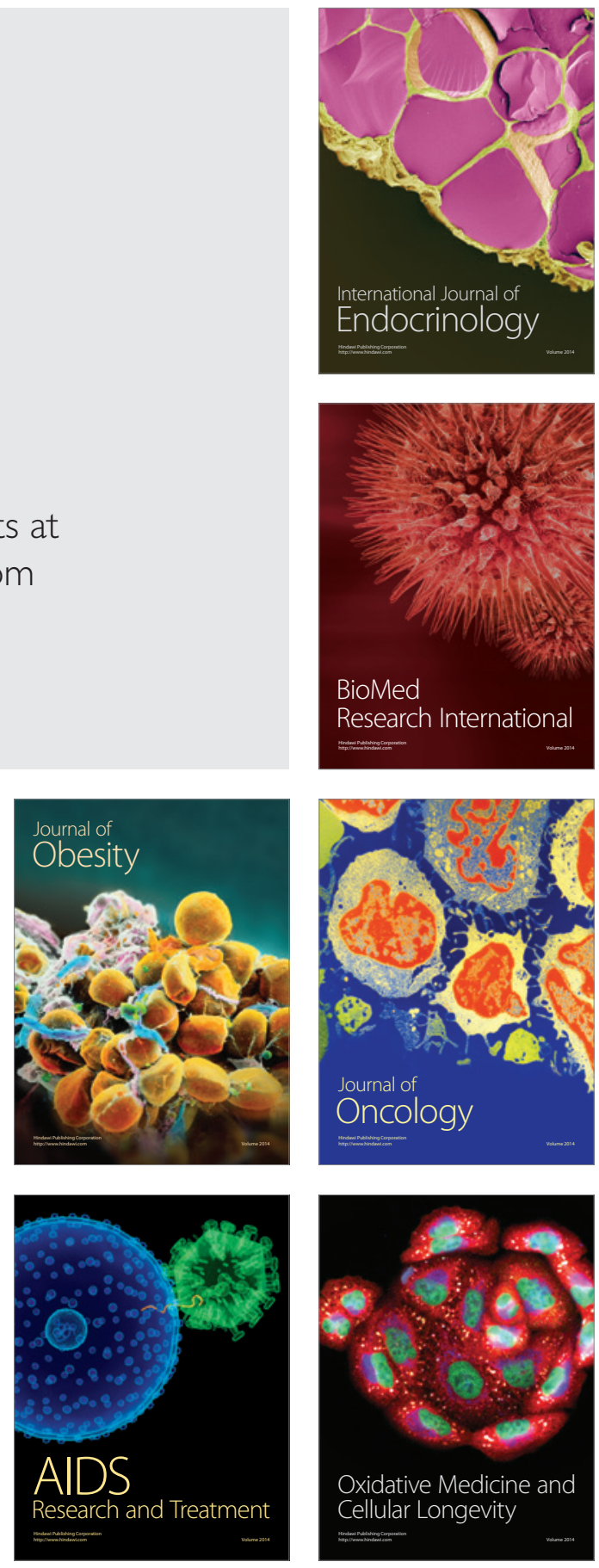